\title{
MACHINE VISION ON-LINE DETECTION QUALITY OF SOFT CAPSULES BASE ON SVM
}

\author{
Fucai Ge ${ }^{1}$, Jiyong $\mathrm{Shi}^{2}$, Youyi $\mathrm{Xu}^{1}$, Xiaobo Zou ${ }^{2, *}$, Jiewen Zhao ${ }^{2}$ \\ 1 Electro-mechinery General Works Co.Ltd, Jiangsu University, Zhenjiang, Jiangsu 212013, \\ China \\ ${ }^{2}$ Agricultural Product Processing and Storage Lab, Jiangsu University, Zhenjiang, Jiangsu \\ 212013, China \\ * Corresponding author, Address: Agricultural Product Processing and Storage Lab, Jiangsu \\ University, 301 Xuefu Road, Zhenjiang, Jiangsu 212013, China, Tel:+86-0511-88780201, \\ Fax:+86-0511-88780201, Email: Zou_xiaobo@ujs.edu.cn
}

Abstract: Nowadays the quality inspect of soft capsules is mainly by manual. Despite the intensive of this work, the accuracy of inspection by manual is very low. This paper proposed soft capsules online sorting system based on machine vision. The inspection process are following: (1) soft capsules were placed on rollers are rotating while moving. The image of each soft capsule was grabbed. (2) automatic threshold based on ostu was used to segmentation capsule image from background, and morphological filter was used to eliminate noise and regional markings. (3) 4 features were extracted which were perimeter, area, girth, altitude diameter and latitude diameter. Support Vector Machine (SVM) and was used to analyze these features. 15460 soft capsules were tested by the online sorting system. The overall grading accuracy was up to $94.1 \%$. Furthermore, the grading speed of the sorting line resches10 capsules per second.

Keywords: $\quad$ Soft Capsules; Machine Vision; On-line Grading

\section{INTRODUCTION}

Soft Capsules is a new kind of capsules in which oil functional material, liquor, suspension mash or even powder is sealed. Soft capsules industry is developing very fast and more than 600 hundred million soft capsules are

Please use the following format when citing this chapter:

Ge, F., Shi, J., Xu, Y., Zou, X. and Zhao, J., 2009, in IFIP International Federation for Information Processing, Volume 294, Computer and Computing Technologies in Agriculture II, Volume 2, eds. D. Li, Z. Chunjiang, (Boston: Springer), pp. 1369-1378. 
produced every year over the world which cost 4 hundred million dollars. There are 3 hundred million soft capsules in our nation each year ,the product is export to Japan, South East Asia, USA, Western Europe, Singapore etc.

As most of contents of Soft Capsules have viscosity, a fraction of content was adhere to injector and filling pump while it flow into wedge injector and was pushed into two pieces of colloidal film by the filling pump of automatic rotating rolling capsules machine(G. Reich et al., 2004).This process caused measurement error and fluctuation of Soft Capsules's weight which has closely correlation to it's efficacy. Therefore Soft Capsules are graded in order to keep their weight uniform. Nowadays, many companies use workers who were trained to judge Soft Capsules weight according their figures subjectively. But the grading accuracy and repeatability was low in that the grading process was based on workers personal experience, also because of the large labor intensity, the expensive cost and the low efficiency, this grading method can not apply to modern produce.

At present, there is not a on-line grading quality equipment for Soft Capsules, so we proposed to use machine vision to resolve the situation and designed a on-line grading system that could classify soft capsules according weight standard.

\section{SYSTEM SETUP OVERVIEW}

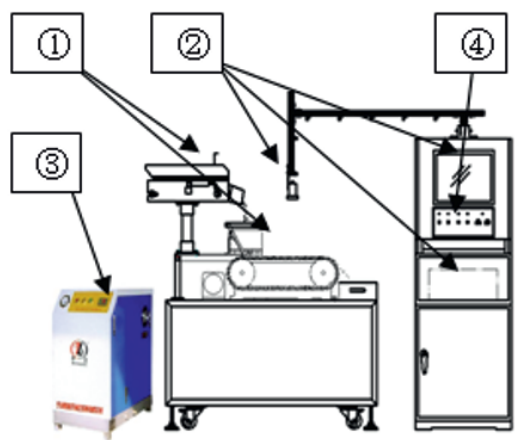

Fig. 1 Soft Capsule online sorting device entire map

A system capable of grading soft capsule at on-line throughput was developed. The setup of the system is show in Fig. 1. It consisted of feeding unit, machine vision system, grading unit and electric control unit. The basic feeding conveyor transported the soft capsule to the uniform spacing conveyor. Then, the capsule were fed to the machine vision system for the defect inspection. Finally, the automatic sorting unit accomplished the soft capsule grading operation. 

SVM

The machine vision system included a lighting chamber for the desired spectrum and light distribution for soft capsule illumination, a CCD camera and an image grabbing card with four input channels which provided by Euresys company inserted in a microcomputer (processor speed: $1.66 \mathrm{GHz}$ ).

\section{IMAGE PROCESS}

\subsection{Image background removal}

There are many ways to remove background of a image (Milan Sonka et al., 2003), according to the histogram, gray distribution of the soft capsule images is double peak. As this distribution we chose ostu maximum variance between clusters(OSTU method) to remove the background. Fig2 (a) is source image and fig 2 (b) is the result image processed by OSTU method, from the image we can get that the soft capsule was segmented completely.

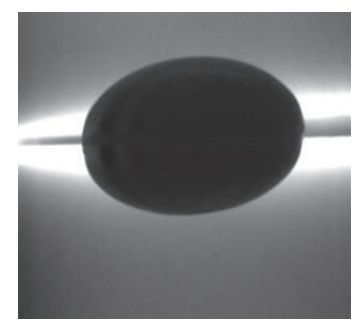

(a)

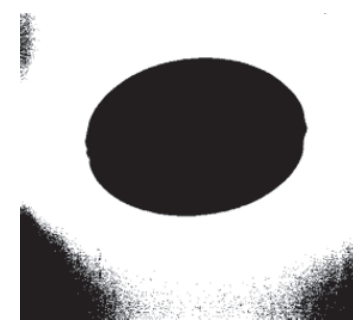

(b)

Fig. 2 Before and after removing the background image Capsule

\subsection{Noise removal}

Although background was removed from source image, the result image still existed some noise which will influence with future processing. There are many methods to remove noise from a image such as mean smoothing, Low-pass Filter and median filter. In the research we choose $3 \times 3$ mean smoothing filter,

Low-pass Filter and median filter to remove noise(Rafael C.Gonzelez Richard et al., 2003) .The results of these method are as follow images in fig 3.Compared their effects, we found

fig 3 (d) was the best image for future processing. 

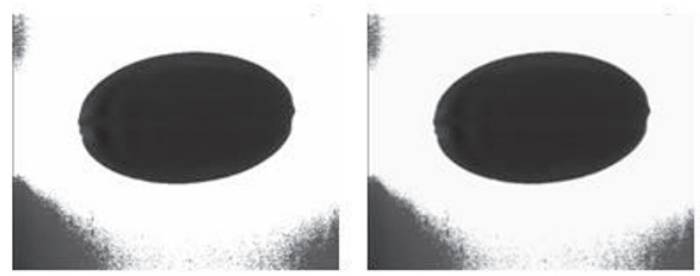

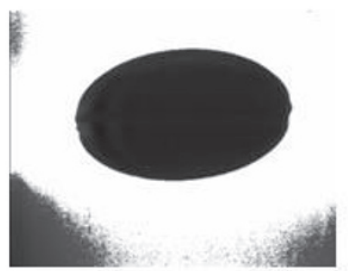

(c)

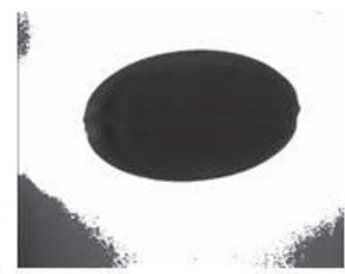

(d)

Fig3 effect of mean smoothing、 Low-pass Filter and median filter

(a)source image (b)processed by mean smoothing

(c)processed by Low-pass Filter (d)processed by median filter

\subsection{Image character extraction}

In order to keep whole soft capsule region in background removing step, some background pixel which has similar gray value were reserved. Before character extraction, we should do region labeling (Rafael C.Gonzelez Richard et al., 2003)to find right region of soft capsule in the image. On this paper, because many machine vision softs have region labeling algorithm so we chose blob analyses function which include in Evision soft to do this work. The result is show in fig4. In the image, we define that a region whose pixels is more than 50000 is soft capsule region.

After the region of soft capsule was found in image, we should extract characters for grading judgement. In this research, we used area, girth, altitude diameter and latitude diameter to represent soft capsule character. Their definition are shown in fig 5.
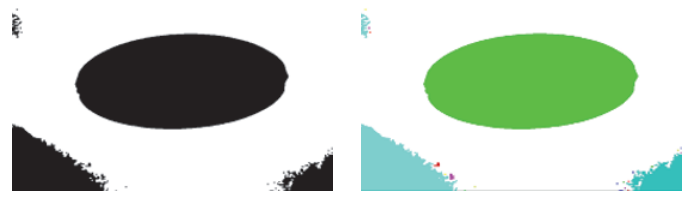

Fig 4 region labeling

(1) area $\mathrm{S}$ as shown in fig5, the number of pixels whose gray value is 0 .

(2) girth L as shown in fig5, the number of the edge of soft capsule region, 
(3) altitude diameter $\mathrm{H}$ the distance between the most left and right pixel.

(4) latitude diameter $\mathrm{W}$ the distance between the most top and bottom pixel

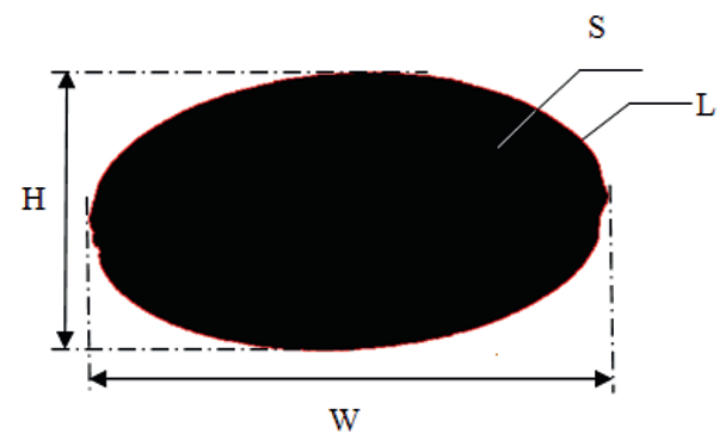

Fig 5 characters of soft capsule

\section{DETECTION QUALITY BY SVM MODEL}

\subsection{Support Vector Machine (SVM)}

Support Vector Machine(SVM) procedure is based on statistical learning theory as proposed by Vapnik and Chervonenkis (V.N. Vapnik, A.Y et al., 1995), which is discussed in detail by Vapnik (V.N. Vapnik, A.Y et al., 1995). The SVM can be seen as a method of training polynomial, radial basis function, or multilayer perception classifiers, in which the weights of the network are found by solving a quadratic programming (QP) problem with linear inequality and equality constraints. The SVM uses structural risk minimization, rather than a non-convex, unconstrained minimization problem, as in standard neural network training technique using empirical risk minimization. Empirical risk minimizes the misclassification error on the training set, whereas structural risk minimizes the probability of misclassifying a previously unseen data point drawn randomly from a fixed but unknown probability distribution(S.R. Gunn et al., 1995).

Assume that the training data with $k$ number of samples is represented by $\left\{\mathrm{x}_{\mathrm{i}}, \mathrm{y}_{\mathrm{i}}\right\}=1,2, \ldots \mathrm{k}$, where $\mathrm{x} \in \mathrm{R}^{\mathrm{n}}$ is an $\mathrm{n}$ dimensional vector and $\mathrm{y} \in\{-1,1\}$ is the class label. These training patterns are said to be linearly separable if a vector $\omega$ and a scalar $\beta$ can be defined so that inequalities (1) and (2) are satisfied: 


$$
\begin{aligned}
& \omega \bullet x_{i}+\beta \geq+1, \text { for all } y=+1 \\
& \omega \bullet x i+\beta \leq-1, \text { for all } y=-1
\end{aligned}
$$

The aim is to find a hyperplane that divides the data so that all the points with the same label are on the same side of the hyperplane. This amounts to finding $\omega$ and $\beta$ such that:

$$
\mathrm{yi}(\omega \bullet \mathrm{xi}+\beta)>0
$$

If a hyperplane exists that satisfies (3), the two classes is said to be linearly separable. In this case, it is always possible o rescale $\omega$ and $\beta$ so that $\min _{1 \leq i \leq k} \operatorname{yi}(\omega \bullet \mathrm{xi}+\beta) \geq 1$.

That is, the distance from the closest point to the hyperplane is $1 /\|\omega\|$. Then (3) can be written as

$$
\mathrm{yi}(\omega \bullet \mathrm{xi}+\beta) \geq 1
$$

The hyperplane for which the distance to the closest point is maximal is called the optimal separating hyperplane (OSH) (Fig. 6). As the distance to the closest point is $1 /\|\omega\|$, the OSH can be found by minimizing $\|\omega\|^{2}$ under constraint (4). The minimization procedure uses Lagrange multipliers and

quadratic programming $(\mathrm{QP})$ optimization methods. If $\alpha i \geq 0, i=1, \ldots, k$ are the non-negative Lagrange multipliers associated with constraint (4), the optimization problem becomes one of maximizing:

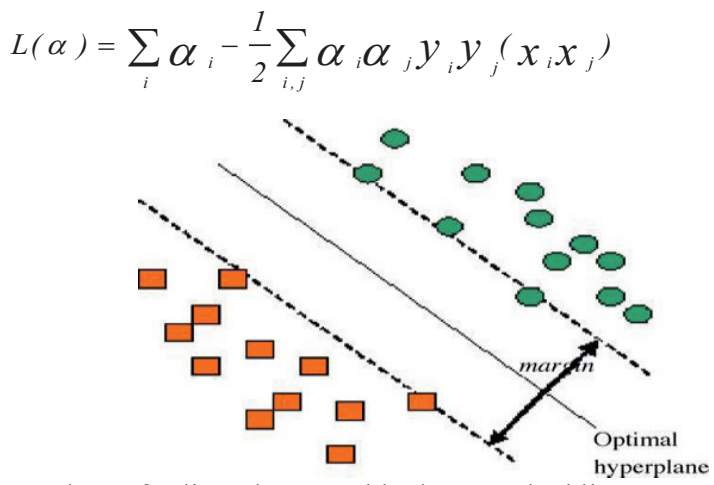

Fig. 6. Hyperplanes for linearly separable data. Dashed line passes through the support vectors

Under constrains: $\alpha i \geq 0, i=1, \ldots, k$. If $\alpha m=(\alpha m 1 \ldots, \alpha m k)$ is an optimal solution of the maximization problem (5) then the optimal separating hyperplane can be expressed as

$$
\omega^{m}=\sum_{i} y_{i} \alpha_{i}^{m} x_{i}
$$


The support vectors are the points for which $\alpha m i>0$ when the equality in (4) holds. If the data are not linearly separable then a slack variable $\xi i, i=1, \ldots, k$ can be introduced with $\xi i=0$ such that (10) can be written as Eq. (7), and the solution to find a generalized OSH, also called a soft margin hyperplane, can be obtained using the conditions: Eqs. (8)-(10):

$$
\begin{aligned}
& y_{i}\left(\omega \bullet x_{i}+\beta\right)-1+\xi_{i} \geq 0 \\
& \min _{\omega, \beta, \xi_{i}, \ldots \xi_{k}}\left[\frac{1}{2}\|\omega\|^{2}+c \sum_{i=1}^{k} \xi_{i}\right] \\
& y_{i}\left(\omega \bullet x_{i}+\beta\right)-1+\xi_{i} \geq 0 \\
& \xi_{i} \geq 0, i=1, \ldots, k
\end{aligned}
$$

The first term in (8) is same as in the linearly separable case to control the learning capacity, while the second term controls the number of misclassified points, while parameter $C$ is chosen by the user. Larger value of $C$ means assigning a higher penalty to errors. In situations in which it is not possible to have a hyperplane defined by linear equations on the training data, the techniques discussed for linearly separable data can be extended to allow for non-linear decision surfaces. A technique introduced by

Vapnik (V.N. Vapnik, A.Y et al., 1995) maps input data into a high dimensional feature space through some non-linear mapping. The transformation to a higher dimensional space spreads the data out in a way that facilitates the finding of linear hyperplane (Fig. 1). After replacing $\mathbf{x}$ by its mapping in the feature space $\Phi(x)$, Eq. (5) can be written as

$$
L(\alpha)=\sum_{i} \alpha_{i}-\frac{1}{2} \sum_{i, j} \alpha_{i} \alpha_{j} y_{i} y_{j}\left(\phi\left(x_{i}\right) \phi\left(x_{j}\right)\right)
$$

It is convenient to introduce the concept of the kernel function $K$, in order to make the computation easier in feature space, such as Eq. (12):

$$
K\left(x_{i}, x_{j}\right)=\phi\left(x_{i}\right) \phi\left(x_{j}\right)
$$

It is the kernel function that performs the non-linear mapping. Popular kernel functions are the following Eqs. (13)-(15):

RBF kernel function : RBF kernel function:

$$
K\left(x_{i}, x_{j}\right)=\exp \left(-\frac{\left\|x_{i}-x_{j}\right\|^{2}}{2 \theta^{2}}\right)
$$

polynomial kernel function : 


$$
K(x i, x j)=(1+x i x j)^{\sigma}
$$

sigmoid kernel function :

$$
(x i, x j)=\tanh (\sigma(x i x j)+v)
$$

Thus, to solve Eq. (5), only the kernel function is computed rather than $\Phi(x)$, which could be computationally expensive. Eq.(16) can be used to classification function:

$$
Y=\operatorname{sign}\left\{\sum_{i, j} \alpha_{i} \alpha_{j} y_{i} y_{j} K\left(x_{i}, x_{j}\right)+\beta\right\}
$$

In brief, SVM firstly maps the data which are not linearly separable into a high dimensional feature space, and then classifies the data by the maximal margin hyperplanes (Fig. 7 ).

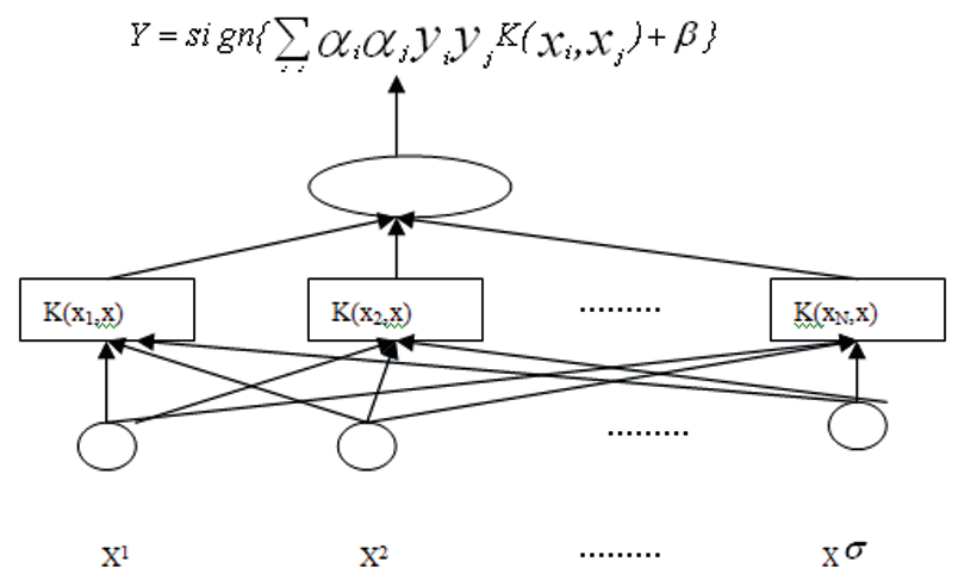

Fig 7 The construction of SVM.

\subsection{Results}

Radial Basis Function was choose to build SVM model to recognize unqualified soft capsules. In order to get strong Generalization Ability of SVM model, The influences of the error penalty parameter $\mathrm{C}$ and the Gaussian kernel parameter $\sigma$ were studied. 540 soft capsules(180 unqualified and 360 qualified) were chose to extract area, girth, altitude diameter and latitude diameter to build SVM. 100 unqualified and 200 qualified in training set, the rest of soft capsules in testing set. According to the accurate rates of training samples and testing samples, the error penalty parameter $\mathrm{C}$ and the Gaussian kernel parameter $\sigma$ can be determined. Results is shown in table 1. The accurate rate of testing set reached highest while $\mathrm{C}=32 \sigma=2$ and the SVM model get strongest Generalization Ability. 
Table 1 Selection of parameter and result of the experiment

\begin{tabular}{lcccccccccccccccccc}
\hline $\begin{array}{l}\text { Parameter } \\
\text { value }\end{array}$ & $\mathrm{C}$ & $\sigma$ & $\mathrm{C}$ & $\sigma$ & $\mathrm{C}$ & $\sigma$ & $\mathrm{C}$ & $\sigma$ & $\mathrm{C}$ & $\sigma$ & $\mathrm{C}$ & $\sigma$ & $\mathrm{C}$ & $\sigma$ & $\mathrm{C}$ & $\sigma$ & $\mathrm{C}$ & $\sigma$ \\
\cline { 2 - 3 } & $2^{-3}$ & $2^{-7}$ & $2^{-1}$ & $2^{-5}$ & $2^{0}$ & $2^{-3}$ & $2^{1}$ & $2^{-1}$ & $2^{3}$ & $2^{0}$ & $2^{5}$ & $2^{1}$ & $2^{7}$ & $2^{3}$ & $2^{9}$ & $2^{5}$ & $2^{11}$ & $2^{7}$ \\
\hline $\begin{array}{l}\text { Accuracy } \\
\text { of training } \\
\text { set (\%) }\end{array}$ & 97.2 & 97.2 & 96.3 & 95.4 & 95.4 & 94.4 & 94.4 & 93.5 & 92.6 \\
$\begin{array}{l}\text { Accuracy } \\
\text { of testing } \\
\text { set (\%) }\end{array}$ & 61.1 & & 87.5 & 90.3 & 93.1 & 94.4 & 91.7 & 90.3 & 86.5 & 93.1 \\
\hline
\end{tabular}

15460 soft capsules produced by Hengshun company were tested by the on-line grading system based on SVM. The accurate rate of grading is show in table 2. The solft capsules were first detected by manual using electronic scale (FA1604), and sorted into two classes: accepted and rejected.

Table. 2 The detection accuracy rate of Capsule by SVM

\begin{tabular}{cccc}
\hline $\begin{array}{c}\text { Total number of } \\
\text { samples }\end{array}$ & $\begin{array}{c}\text { Number of } \\
\text { Accepted }\end{array}$ & $\begin{array}{c}\text { Number of } \\
\text { Rejected }\end{array}$ & $\begin{array}{c}\text { Accuracy of detection } \\
\text { by SVM (\%) }\end{array}$ \\
\hline 15460 & 14547 & 913 & $94.1 \%$ \\
\hline
\end{tabular}

The detection results of SVM is $94.1 \%$ as shown in table 2. Compared with the manual detection by huaman eyes (the accurate rate of detection is $84.9 \%$ ), the machine detection with SVM is much higher.

\section{CONCLUSION}

The quality of soft capsules online detection system based on machine vision was developed in this paper. SVM is discussed and used to distinguish the rejected capsule from accepted ones. The image of each soft capsule was grabbed from the detection line when they are rotating while moving. Automatic threshold based on ostu was used to segmentation capsule image from background, and morphological filter was used to eliminate noise and regional markings. 4 features were extracted which were perimeter, area, girth, altitude diameter and latitude diameter. Support Vector Machine (SVM) and was used to analyze these features. 15460 soft capsules were tested by the online sorting system. The overall grading accuracy was up to $94.1 \%$. Furthermore, the detection speed of the sorting line resches 10 capsules per second.

The results can be used in soft capsule Production Line to inspect the quality of soft capsules accurately and rapidly. It fills the bank of soft capsule on-line grading technology in the industry. Furthermore, this system can be used to grade other productions. 


\section{ACKNOWLEDGEMENTS}

It is gratefully acknowledged that this work is supported by the National High Technology Research and Development Program of China and National Natural Fund of China.

\section{REFERENCES}

G. Reich, Formulation and physical properties of soft capsules, in: F. Podczek, B.E. Jones (Eds.), Pharmaceutical Capsules, Pharmaceutical Press, London, 2004, pp. 201-212.v

Milan Sonka. Image Processing Analysis and Machine Vision[M]. Beijing. Posts \& Telecom Press. 2003

Rafael C.Gonzelez Richard, Digital Image Processing [M].Beinjing :Publishing house of electronics industry,2003

S.R. Gunn, Support Vector Machines for Classification and Regression. Technical Report: Image Speech and Intelligent Systems Research Group. Paper available on http://www.isis.ecs.soton.ac.uk/ resources/svminfo/.

V.N. Vapnik, A.Y. Chervonenkis, Theory Prob. Appl. 17 (1971) 264.[18] V.N. Vapnik, The Nature of Statistical Learning Theory, Springer-Verlag, New York, 1995.

V.N. Vapnik, The Nature of Statistical Learning Theory, Springer-Verlag,New York, 1995. 